\title{
Impact of surface dynamo magnetic fields on the solar abundance of the CNO elements
}

\author{
N. Shchukina ${ }^{1,2}$, A. Sukhorukov ${ }^{2,5}$, and J. Trujillo Bueno ${ }^{1,3,4}$ \\ 1 Instituto de Astrofísica de Canarias, 38205 La Laguna, Tenerife, Spain \\ 2 Main Astronomical Observatory, National Academy of Sciences, 27 Zabolotnogo Street, 03680 Kiev, Ukraine \\ e-mail: shchukin@mao.kiev.ua \\ 3 Departamento de Astrofísica, Universidad de La Laguna, 38206 La Laguna, Tenerife, Spain \\ ${ }^{4}$ Consejo Superior de Investigaciones Científicas, Spain \\ 5 Institute for Solar Physics, Department of Astronomy, Stockholm University, AlbaNova University Centre, 10691 Stockholm, \\ Sweden
}

Received 2 May 2015 / Accepted 2 November 2015

\section{ABSTRACT}

\begin{abstract}
Most studies of the solar metallicity, based on abundance determinations of the CNO elements, ignore the fact that the quiet solar photosphere is significantly magnetized by a small-scale magnetic field with a mean field strength of $\sim 100$ G. Here we quantify how this significant magnetization affects determinations of the abundances of these chemical elements. To this end, we used two three-dimensional models of the solar photosphere taken from a magneto-convection simulation with small-scale dynamo action, one virtually unmagnetized, and the other characterized by a mean field strength of $160 \mathrm{G}$ in the low photosphere. We performed local thermodynamic equilibrium spectral synthesis for a large set of C I, N I, and O I lines to derive abundance corrections. We included the magnetic broadening of the lines (direct effect) and the magnetically induced changes of the photospheric temperature stratification (indirect effect). We find that these small-scale dynamo magnetic fields only negligibly affect the determination of the solar abundances of carbon, nitrogen, and oxygen.
\end{abstract}

Key words. dynamo - convection - line: formation - magnetohydrodynamics (MHD) - Sun: abundances - radiative transfer

\section{Introduction}

The application of three-dimensional (3D) hydrodynamical (HD) models to determining the abundances of $\mathrm{C}, \mathrm{N}, \mathrm{O}$ and other elements such as Fe and Si (e.g., Asplund 2000; Asplund et al. 2000, 2005a,b, 2009; Caffau et al. 2011; Pereira et al. 2013; Scott et al. 2015, and references therein) has led to a downward revision of the solar metallicity from $Z / X=0.0275$ (Anders \& Grevesse 1989) to $Z / X=0.0165$ (Asplund et al. 2005b) and later to slightly higher values: $Z / X=0.0181$ (Asplund et al. 2009) and $Z / X=0.0209$ (Caffau et al. 2011). These revised values deviate from the standard solar metallicity $Z / X=0.0231$ (Grevesse \& Sauval 1998), which resulted in a disagreement between solar interior models and helioseismological data (e.g., Bahcall et al. 2005; Basu \& Antia 2008; Serenelli et al. 2009). We note that of the chemical elements listed above, carbon, nitrogen, and oxygen play a crucial role in determining the solar metallicity because they contribute more than $60 \%$ to the $Z / X$-value.

All the above-mentioned chemical abundance investigations used unmagnetized models of the solar atmosphere. However, the quiet solar photosphere is significantly magnetized by a complex, small-scale magnetic field, which according to Trujillo Bueno et al. (2004) has a mean field strength of $\sim 100 \mathrm{G}$ (see also the reviews by Trujillo et al. 2006; Sánchez Almeida \& Martínez González 2011; Stein 2012; Martínez Pillet 2013; Stenflo 2013). For this reason, it is important to investigate whether this significant magnetization may affect the determination of the chemical abundances.
The presence of a magnetic field can affect spectral lines both directly (through Zeeman broadening) and indirectly (through the magnetically induced changes of the thermodynamical structure of the atmosphere).

Using one-dimensional (1D) semi-empirical models of the quiet solar photosphere, Borrero (2008) investigated the direct impact of magnetic fields on the abundances of $\mathrm{Fe}, \mathrm{Si}, \mathrm{C}$, and $\mathrm{O}$. He concluded that for silicon, carbon and oxygen the direct impact of magnetic fields is very marginal (with abundance corrections smaller than 0.02 dex).

Fabbian et al. (2012) investigated the direct and indirect impact of small-scale magnetic fields following a more realistic approach. They used 3D models of the solar photosphere taken from magneto-convection simulations characterized by non-zero values of the net magnetic flux (their simulations were initialized using externally imposed vertical magnetic fields) and concluded that a magnetization of about $100 \mathrm{G}$ would have a significant effect (of about $0.1 \mathrm{dex}$ ) on the inferred iron abundance. Fabbian et al. (2012) also concluded that the magnetic fields of their 3D models have a similar effect on the equivalent widths of the following lines: the $\mathrm{C}$ I line at $4775.90 \AA$, the O I line at $6158.18 \AA$, the Ni I line at $6767.77 \AA$, the Mn I at $8740.91 \AA$, and the Si I line at $10827 \AA$.

Shchukina \& Trujillo Bueno (2015) recently argued that a more realistic representation of the small-scale magnetic activity of the quiet solar photosphere is given by 3D magnetoconvection snapshots with a mean field strength of about $100 \mathrm{G}$, but with zero net magnetic flux. These authors used two 3D snapshot models taken from Rempel's (2014) small-scale 
dynamo (SSD) numerical experiments (where the net magnetic flux is zero), one practically unmagnetized and the other characterized by a mean field strength of $160 \mathrm{G}$ in the low photosphere. Shchukina \& Trujillo Bueno (2015) analyzed in detail a carefully chosen set of $66 \mathrm{Fe}$ I lines and concluded that if the small-scale magnetization of the quiet solar photosphere is similar to the one in Rempel's (2014) model, then its effect on the determination of the iron abundance is negligible. A similar conclusion has independently been obtained by Moore et al. (2015) for both iron (using four Fe I lines) and oxygen (using five $\mathrm{O}$ I lines).

The aim of this paper is to extend the above-mentioned Fe I investigation by Shchukina \& Trujillo Bueno (2015) to the CNO elements, explaining in detail the results we summarized in Shchukina et al. (2015). Section 2 describes the 3D photospheric models used in this study and the $\mathrm{C} \mathrm{I}, \mathrm{N}$ I, and $\mathrm{O}$ I line data, along with the strategy used for the line spectral synthesis. In Sect. 3 we present the derived differential abundance corrections, and in Sects. 4 and 5 we summarize the main conclusions.

\section{Input data and method}

\subsection{D MHD model}

We used two 3D snapshot models resulting from the magnetoconvection simulations with small-scale dynamo action carried out by Rempel (2014). To facilitate the radiative transfer calculations, the original snapshots with a resolution of $8 \times 8 \times 8 \mathrm{~km}$ were interpolated to a coarser resolution grid with $80 \mathrm{~km}$ and $8 \mathrm{~km}$ in the horizontal and vertical directions, respectively. This corresponds to a grid of $77 \times 77 \times 126$ points instead of the $768 \times 768 \times 384$ original grid. The $\mathrm{C}, \mathrm{N}, \mathrm{O}$ abundance corrections were found to be insensitive to this reduction of the horizontal resolution. A more detailed description of the snapshots used can be found in Shchukina \& Trujillo Bueno (2015). We recall that the first snapshot model, with a vertical unsigned flux density $\left\langle\left|B_{z}\right|\right\rangle=0.5 \mathrm{G}$ in the lower photosphere, represents the kinetic growth phase. The mean field strength $\langle B\rangle$ of this model is very close to zero. The second snapshot model with $\left\langle\left|B_{z}\right|\right\rangle=80 \mathrm{G}$ corresponds to the stationary stage. Shchukina \& Trujillo Bueno (2015) showed that the $\left\langle\left|B_{z}\right|\right\rangle=80 \mathrm{G}$ snapshot is similar to other snapshots from the stationary stage of Rempel's (2014) magneto-convection simulation, while the $3 \mathrm{D}$ snapshot model with $\left\langle\left|B_{z}\right|\right\rangle=0.5 \mathrm{G}$ is very similar to those resulting from his purely hydrodynamical simulation, at least in the model photosphere (i.e., until a height of $500 \mathrm{~km}$ above the visible model surface). We point out that all the $\mathrm{C}$ I, N I, and $\mathrm{O}$ I lines we used originate approximately between $0 \mathrm{~km}$ and $200 \mathrm{~km}$ (i.e., at heights with continuum optical depths $\log _{10} \tau_{5}<-1$ ). This gives us confidence that our investigation of the abundance corrections based on the use of the two snapshots of the Rempel (2014) simulations is reliable.

According to Shchukina \& Trujillo Bueno (2015), the mean temperatures of the snapshot models by Rempel (2014) in the lower photosphere $\left(\log _{10} \tau_{5}<-0.5\right)$ agree very well with the temperature of the semi-empirical model MACKKL (Maltby et al. 1986), which is based on observations of the continuum radiation. As a result, the absolute continuum intensities computed at different wavelengths in Rempel's 3D models and in the MACKKL 1D model are consistent with observations. This agreement can be interpreted as evidence that the snapshot is realistic, at least in the continuum forming layers. Above $\log _{10} \tau_{5}>-0.5$ the 3D snapshot models of Rempel (2014) become significantly cooler than the MACKKL $1 \mathrm{D}$ model. Testing the reliability of the $3 \mathrm{D}$ models in these higher layers would require spectral synthesis and comparison with other observational data, such as the center-to-limb variation of the absolute intensity, the intensities at the wings of the H I Balmer lines, and the asymmetries and shifts of unblended lines. Such comparisons are beyond the scope of the present paper. Our goal here is to estimate differential abundance corrections caused by small-scale dynamo photospheric magnetic fields; to this end, it is sufficient to use the two 3D snapshot models obtained from Rempel's (2014) simulations.

\subsection{Line parameters and data analysis}

The list of the CI, N I, and O I lines used for estimating the impact of SSD magnetic fields on the abundance determinations is given in Table 1. For most of the lines, we used "solar" oscillator strengths $(\log g f)$ and observed equivalent widths $(W)$ from Gurtovenko \& Kostik (1989). They are indicated in Table 1 with the letters "GK". The remaining lines have $W$ and $\log g f$ values from Asplund et al. (2004, 2005b), Caffau et al. (2010), and Lambert (1978). They are indicated by "A04", "A05", "C", and "L". The effective Landé factors $\left(g_{\mathrm{L}}\right)$ were calculated using the LS coupling formula.

We assumed Voigt profiles for the absorption line shapes of $\mathrm{CI}, \mathrm{N}$ I, and OI. The line damping was derived as the sum of Van der Waals and radiative broadening. The main contribution is given by Van der Waals broadening by $\mathrm{H} \mathrm{I}$ and $\mathrm{He} \mathrm{I}$ atoms and $\mathrm{H}_{2}$ molecules. The Van der Waals broadening constant $\gamma_{6}$ was determined using the classical approximation (see Eq. (82.48)) of Unsöld (1955) with the enhancement factor $E=1.5$ recommended by Gurtovenko \& Kostik (1989). Since we estimate here only differential abundance effects, the choice of the enhancement factor does not affect our results (see also Fabbian et al. 2012).

We obtained the line intensity profiles at the solar disk center by solving the radiative transfer equation for all the spectral lines of Table 1 and for each vertical 1D column of the 3D snapshots under consideration. The spectral synthesis was performed using the local thermal equilibrium (LTE) approximation. The resulting $\mathrm{CI}, \mathrm{N}$ I, and $\mathrm{O}$ I line intensities were spatially averaged. We calculated the line equivalent widths $W$ for several values of the abundances $A_{\mathrm{C}}, A_{\mathrm{N}}$, and $A_{\mathrm{O}}$. For each C I, N I, and $\mathrm{O}$ I line under consideration, the final value of the abundance corresponding to the observed equivalent width was determined by interpolating the intermediate equivalent widths obtained using varying abundances.

We derived abundance corrections $\Delta A$ accounting for the direct and indirect impact of the small-scale dynamo magnetic fields on the solar abundance determination of $\mathrm{CNO}$ elements $\Delta A=A\left(\left\langle\left|B_{z}\right|\right\rangle=80 \mathrm{G}\right)-A\left(\left\langle\left|B_{z}\right|\right\rangle=0.5 \mathrm{G}\right)$ between the abundances fitted using the snapshots with $\left\langle\left|B_{z}\right|\right\rangle=80 \mathrm{G}$ and $\left\langle\left|B_{z}\right|\right\rangle=0.5 \mathrm{G}$. The indirect effect was found using the same snapshots, but assuming zero magnetic field at each spatial point. We estimated the direct impact of the surface dynamo magnetic fields by calculating the abundance changes $\Delta A=A(B \neq$ $0)-A(B=0)$ caused by decreasing of the magnetic field to zero in the $\left\langle\left|B_{z}\right|\right\rangle=80 \mathrm{G}$ snapshot.

We also calculated the mean formation heights of the $\mathrm{C}$ I, N I, and $\mathrm{O}$ I lines. To this end, we used the concept of the EddingtonBarbier height of formation, meaning that for each surface point of the snapshot under consideration, we computed the optical depth $\tau_{5}$ at $5000 \AA$ where the line optical depth at any given line wavelength point $\Delta \lambda$ equals unity: $\tau_{\Delta \lambda}=1$. With this definition, 
N. Shchukina et al.: Impact of surface dynamo magnetic fields on the CNO abundances
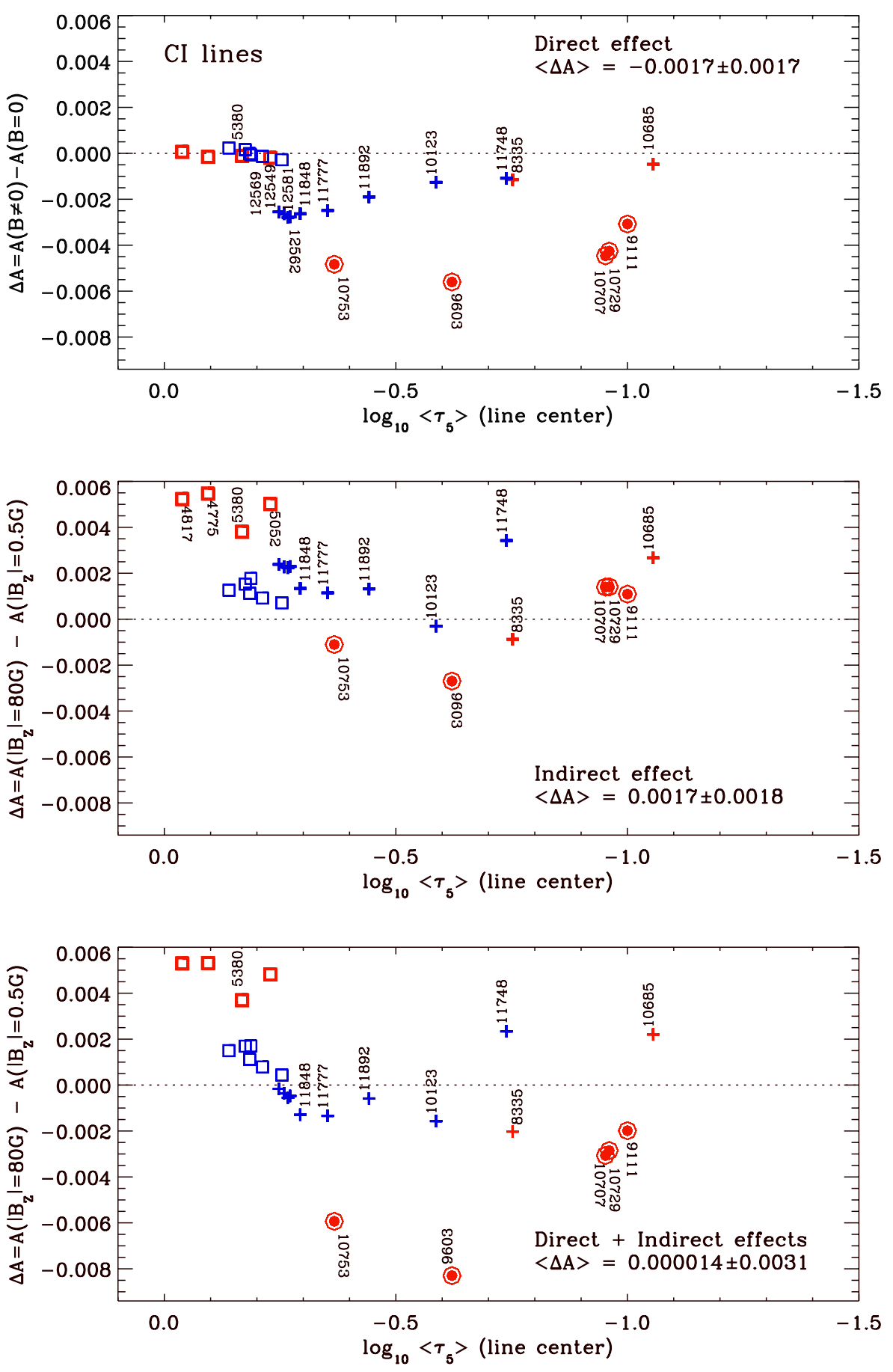

Fig. 1. Abundance corrections caused by the direct (top), indirect (middle), and joint (bottom) actions of the surface dynamo magnetic fields on the determination of the solar carbon abundance. The joint effect represents the sum of $\Delta A$ values shown in the top and middle panels. The abundance corrections are given against the mean line-center optical depths of the C I lines. The mean of the abundance corrections $\langle\Delta A\rangle$ and the corresponding r.m.s. values are shown in the right corner of each panel. Our results for the weak $\mathrm{C}$ I lines are indicated by squares. Crosses and circles represent moderate and strong lines. For the same formation heights, the lines marked by crosses have approximately twice lower $\Delta \lambda_{\langle B\rangle}$ values than the lines marked by circles. Red and blue symbols show the lines with the EPL $<7.5 \mathrm{eV}$ and $\mathrm{EPL}>8.5 \mathrm{eV}$. the mean formation height $\left\langle\tau_{5}\right\rangle$ of the individual lines represents the averaging over a $77 \times 77$ array of derived $\tau_{5}$-values.

\section{Results}

\subsection{Impact of solar surface dynamo magnetic fields on the carbon abundance determination}

Figure 1 shows the impact of the SSD magnetic fields of Rempel's (2014) model on the carbon abundance determined using the $\mathrm{C}$ I lines from Table 1 . The top, middle, and bottom panels of this figure display the abundance corrections $\Delta A$ caused by the direct, indirect, and by both effects. We note that in this paper the $\Delta A$ values for each of the lines under consideration are given as a function of the mean optical depth ( $\tau_{5 \text { (line center) }}$, at $5000 \AA$ ) where the optical depth at the center of the lines is unity. The latter values were calculated using the $\left\langle\left|B_{z}\right|\right\rangle=0.5 \mathrm{G}$ snapshot.

The top panel of Fig. 1 shows that the direct Zeeman broadening effect depends on the formation height of the C I lines (hence, on the equivalent widths) and on their magnetic sensitivity $\Delta \lambda_{\langle B\rangle}=4.67 \times 10^{-10} g_{\mathrm{L}} \lambda^{2}\langle B\rangle$ (where $\langle B\rangle$ is the mean field strength at the optical depth $\left.\left\langle\tau_{5 \text { (line center) }}\right\rangle\right)$. The weak lines indicated in Fig. 1 by squares are, in fact, insensitive to the magnetic Zeeman broadening effect. As a result, the abundance corrections $\Delta A$ for them are very small. These lines have equivalent widths $W<41 \mathrm{~m} \AA, \Delta \lambda_{\langle B\rangle}<5.5 \mathrm{~m} \AA$ and are formed rather deep down, that is, below $\log \left\langle\tau_{5 \text { (line center) }}\right\rangle \approx-0.25$. The $\Delta \lambda_{\langle B\rangle}$-values of strong and moderately strong $\mathrm{C}$ I lines (i.e., the lines with $W>41 \mathrm{m \AA}$ ), marked by crosses in Fig. 1, decrease from $17 \mathrm{~m} \AA$ 
till $2.4 \mathrm{~m} \AA$ in the range $-1.05 \leq \log \left\langle\tau_{5 \text { (line center) }}\right\rangle \leq-0.25$. As a consequence, the abundance corrections for these lines decrease with their formation height. For the same formation heights, the magnetic sensitivity of the lines indicated with circles in Fig. 1 is approximately twice as high as the lines marked by crosses. Therefore, the direct effect for them is stronger ( $\Delta A<-0.003 \mathrm{dex})$. Of these lines, the two lines with an effective Landé factor $g_{\mathrm{L}}=2$ (i.e., the C I lines at $9603.0 \AA$ and $10753.9 \AA$ ) are particularly sensitive to the Zeeman broadening effect $(\Delta A \approx-0.005$ dex). We note that negative values of the abundance corrections indicate that abundance determinations neglecting the Zeeman broadening of the $\mathrm{C}$ I lines overestimate the $A_{\mathrm{C}}$-value. On average, the direct impact of the small-scale dynamo magnetic fields on the determination of the solar carbon abundance is small. We find that the mean of the abundance corrections for the $\mathrm{CI}$ lines used in our study is only $\langle\Delta A\rangle=-0.0017 \pm 0.0017$ dex.

The middle panel of Fig. 1 shows that the magnitude of the indirect effect depends on the formation height of the C I lines and on their excitation potential EPL. The weak $\mathrm{CI}$ lines $(W<41 \mathrm{m \AA})$ with EPL $<7.5 \mathrm{eV}$ (red squares) formed in the deepest photospheric layers $\left(\log \left\langle\tau_{5 \text { (line center) }}\right\rangle \leq-0.25\right)$ are much more sensitive to the indirect impact of the SSD magnetic fields than the lines that are formed in the same layers, but have EPL $>8.5 \mathrm{eV}$ (blue squares). For the strong and moderately strong $\mathrm{C}$ I lines ( $W>41 \mathrm{~m} \AA$ ), the dependence of the abundance corrections on the formation height is also different for the lines with EPL $<7.5 \mathrm{eV}$ and EPL $>8.5 \mathrm{eV}$. In the latter case, the $\langle\Delta A\rangle$ values are mostly positive, while in the former case they change sign with height in the model atmosphere. This height dependence of the abundance corrections results from the change with height of the temperature differences between the two 3D snapshot models used (see Figs. 1 and 8 in Shchukina \& Trujillo Bueno 2015). In summary, the mean error of the carbon abundance determinations that ignore the indirect impact of the SSD magnetic fields remains negligible: $\langle\Delta A\rangle=+0.0017 \pm 0.0018$ dex.

The bottom panel of Fig. 1 shows the joint (direct+indirect) action of the SSD magnetic fields on the determination of the solar carbon abundance. The ensuing $\Delta A$-values are given in Table 1. Since the direct and indirect effects for the most of the $\mathrm{C}$ I lines act in opposite ways, the cumulative action of both of them reduces the abundance corrections. Nevertheless, there are two lines with a high effective Landé factor for which the direct and indirect effects operate in the same way. They are the C I 9603.0 ̊ and C I 10753.9 ̊ lines. As a result, abundance corrections for them increase in size, reaching values of $-0.008 \mathrm{dex}$ and -0.006 dex, respectively.

Summarizing, we conclude that the mean of the $\langle\Delta A\rangle$ errors for the $\mathrm{C}$ I lines caused by neglecting the magnetic Zeeman line broadening and the magnetically induced changes of the photospheric thermodynamic stratification are similar in magnitude, but of opposite sign. This means that the impact of the magnetic fields, which is present in the 3D magneto-convection simulation by Rempel (2014), on the carbon abundance determination can be neglected: $\langle\Delta A\rangle=0.0 \pm 0.003$ dex.

\subsection{Impact of solar surface dynamo magnetic fields on the nitrogen abundance determination}

Our results for the nitrogen abundance errors caused by neglecting the impact of the SSD magnetic fields are presented in Fig. 2 and Table 1. The number of clean Ni lines suitable for determining the solar nitrogen abundance is limited. Table 1 shows that all of them are in the near-infrared part of the spectrum; their equivalent widths do not exceed $12 \mathrm{~m} \AA$, and as a consequence, they are formed around the same deep photospheric layers, i.e. $-0.2 \leq \log \left\langle\tau_{5 \text { (line center) }}\right\rangle<-0.1$.

By comparing the $\Delta A$-values shown in the top panels of Figs. 1 and 2, we see that the weak N I lines, like the weak C I lines, are virtually insensitive to the Zeeman broadening effect, notwithstanding their higher magnetic sensitivity that is due to their larger wavelengths: $5.2<\Delta \lambda_{\langle B\rangle}<8.8 \mathrm{m \AA}$. On average, the mean of the $\Delta A$-values for the $\mathrm{N}$ I lines is even lower than for the similar subset of the $\mathrm{C}$ I lines: $\langle\Delta A\rangle=-0.0004 \pm 0.0001 \mathrm{dex}$.

The middle panel of Fig. 2 shows some sensitivity of the weak N I lines to the indirect effect caused by changes of the thermodynamical properties of the solar photosphere. As in the case of the weak C I lines, this occurs because of the increased temperature sensitivity of the high-excitation lines $($ EPL $>10.3 \mathrm{eV})$. In deep photospheric layers, where the weak $\mathrm{C} \mathrm{I}$ and $\mathrm{N} \mathrm{I}$ lines are formed, the 3D snapshot model with $\left\langle\left|B_{z}\right|\right\rangle=$ $80 \mathrm{G}$ is cooler than that with $\left\langle\left|B_{z}\right|\right\rangle=0.5 \mathrm{G}$ (Shchukina \& Trujillo Bueno 2015). The ensuing relative changes of the equivalent widths imply that the $\Delta A$ values become positive. However, the mean error of the nitrogen abundance determination in the latter case is still small: $\langle\Delta A\rangle=+0.002 \pm 0.0005$ dex.

Table 1 and the bottom panel of Fig. 2 show results of the joint (direct and indirect) impact of the SSD magnetic fields on the nitrogen abundance determination. Since the corresponding $\Delta A$ values have opposite sign, the final value of the mean error becomes even lower: $\langle\Delta A\rangle=+0.0016 \pm 0.0006$ dex.

\subsection{Impact of solar surface dynamo magnetic fields on the oxygen abundance determination}

The oxygen abundance errors arising from the neglect of the influence of the SSD magnetic fields are shown in Fig. 3 and Table 1. Similarly as in the case of the N I lines, there is only a small number of relatively clean (unblended) O I lines useful for determining the solar oxygen abundance. First of all, we have five weak lines $(W<6 \mathrm{~m} \AA)$ in the visible part of the solar spectrum $(5577.3 \leq \lambda \leq 6363.7 \AA)$ that form in the deep photospheric layers, below $\log \left\langle\tau_{5 \text { (line center) }}\right\rangle<-0.15$. We indicate them in Fig. 3 by squares. The set of O I lines shown in this figure by circles includes six infrared lines (7771.9 $\leq \lambda \leq 11302.2 \AA)$, five of which are of moderate strength $(30<W<80 \mathrm{m \AA})$, while only the O I $11302.2 \AA$ line is relatively weak $(W=14 \mathrm{~m} \AA)$. They are formed in the higher photospheric layers $(-0.6<$ $\log \left\langle\tau_{5 \text { (line center) }}\right\rangle<-0.16$ ).

The top panel of Fig. 3 shows that the direct impact of the SSD magnetic fields on weak optical O I lines is extremely small, whereas the $\mathrm{O} I$ infrared lines show a higher sensitivity to the Zeeman broadening effect. For these lines, the direct effect increases with the effective Landé factor $g_{\mathrm{L}}$. The largest effect reaches maximum $(\Delta A \approx-0.005$ dex $)$ for the O I $7774.1 \AA$ line with the largest $g_{\mathrm{L}}=1.917$.

The weak OI optical lines, similar to the weak CI and NI lines, are most sensitive to the indirect impact of the SSD magnetic fields (see middle panel of Fig. 3). The $\Delta A$ values for four of them are positive and around $\approx+0.005 \mathrm{dex}$, while the O I $5577.3 \AA$ line shows a smaller effect. The sign of the abundance corrections for the infrared OI lines changes from positive to a negative value with increasing formation height. The $\Delta A$-values for the latter lines are between $\sim+0.0025 \mathrm{dex}$ and $\sim-0.002$ dex. Again, for the O I lines, this variation with height 
N. Shchukina et al.: Impact of surface dynamo magnetic fields on the CNO abundances
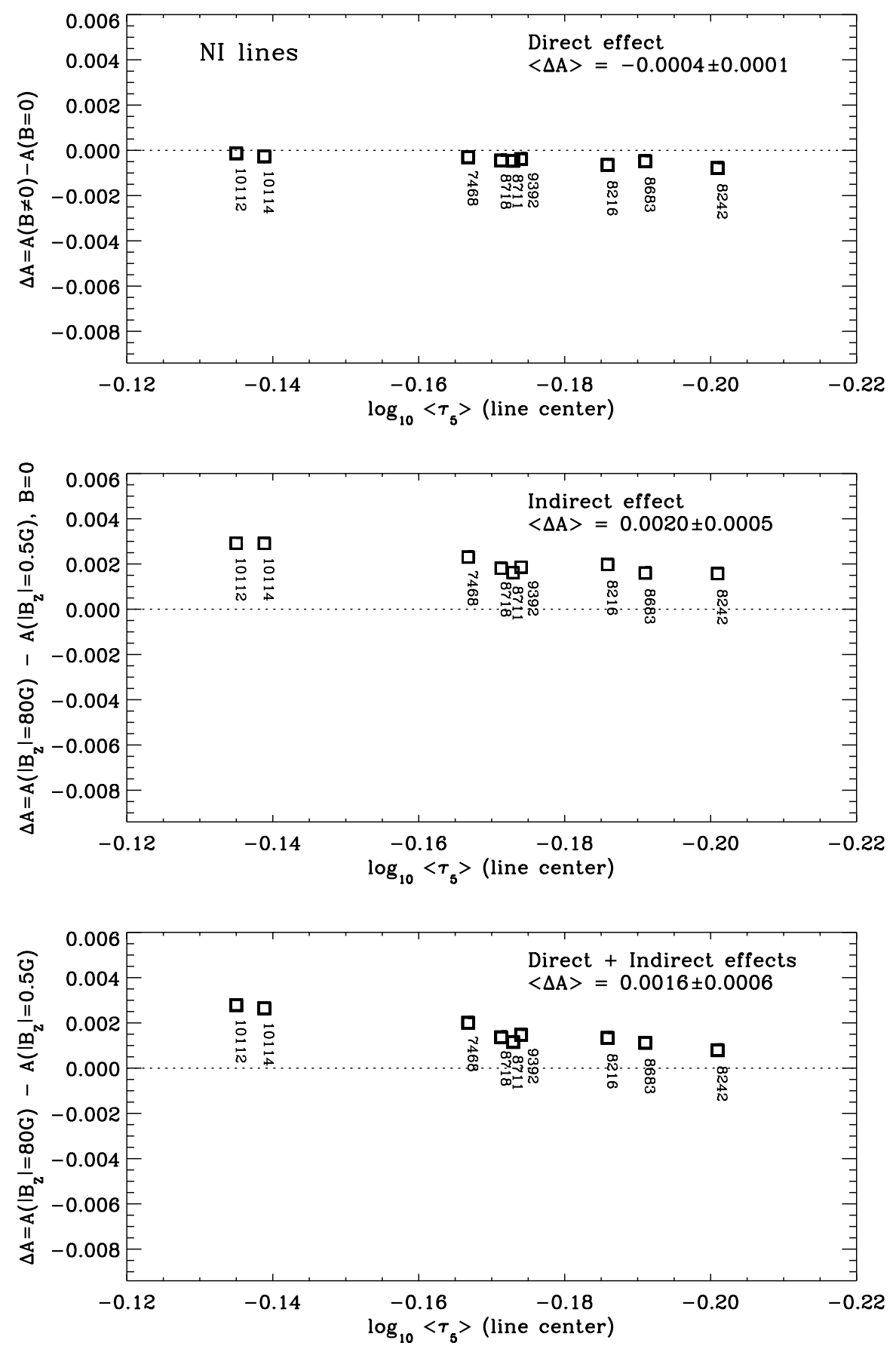

Fig. 2. Same as Fig. 1, but for the solar nitrogen abundance.

of the abundance corrections is related with height changes of temperature differences between the two 3D photospheric models we used.

Overall, the mean values of the oxygen abundance corrections caused by the direct and indirect effects are very similar, while the magnitude of these corrections is very small, $\langle\Delta A\rangle=-0.0018 \pm 0.0018 \mathrm{dex}$ and $\langle\Delta A\rangle=+0.0017 \pm 0.0031 \mathrm{dex}$, respectively. As a result, the mean of the $\langle\Delta A\rangle$ corrections for the $\mathrm{O}$ I lines caused by ignoring the Zeeman line broadening and the magnetically induced changes of the photospheric thermodynamic stratification (see bottom panel of Fig. 3) is very small: $\langle\Delta A\rangle=-0.0001 \pm 0.0048$ dex .

\section{Discussion}

To explain why the CI, NI, and OI lines have these small abundance corrections and, in addition, why the corrections can change their sign, we quantified the intensity profile differences caused by the impact of the SSD magnetic fields. We also visualized how the temperature of the formation layers is connected with these impact changes across the line profiles. Figure 4 shows results for the representative lines. The top left panel of this figure displays changes $\langle\Delta D\rangle_{\Delta \lambda}$ of the line depth $\langle D\rangle_{\Delta \lambda}=1-\langle I\rangle_{\Delta \lambda} /\langle I\rangle_{c}$ across the spatially averaged profiles of the CI 5380.3, C I 9603.0, and C I $10685.3 \AA$ lines by the Zeeman broadening. Here $\langle I\rangle_{\Delta \lambda}$ and $\langle I\rangle_{c}$ are the emerging intensity at a given $\Delta \lambda$-point of the profile and at the continuum. The $\langle\Delta D\rangle_{\Delta \lambda}$ differences of these lines that are due to the indirect impact of the SSD fields presented in the simulations by Rempel (2014) are shown in the top right panel of the same figure. The two bottom panels illustrate the relative changes in the mean temperature $\langle T\rangle_{\Delta \lambda} /\langle T\rangle_{\mathrm{c}}$ of the atmospheric layers where the line intensity $\langle I\rangle_{\Delta \lambda}$ is formed, due to the Zeeman broadening 

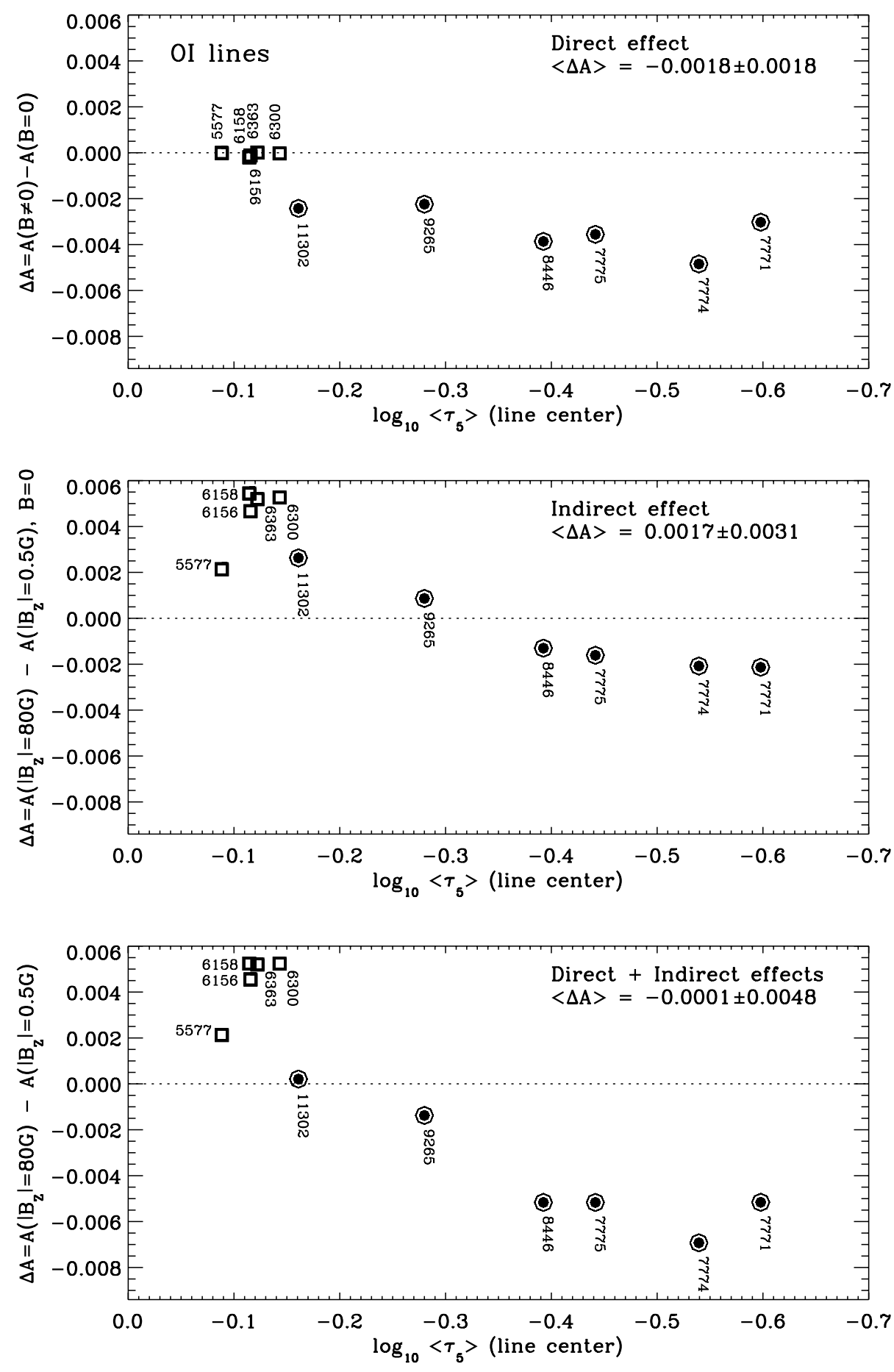

Fig. 3. Same as Fig. 1, but for the solar oxygen abundance.

(left) and to the differences between the temperature structure of the $\left\langle\left|B_{z}\right|\right\rangle=0.5 \mathrm{G}$ and $\left\langle\left|B_{z}\right|\right\rangle=80 \mathrm{G}$ models (right). We note that $\langle T\rangle_{\mathrm{c}}$ is the mean temperature of the photospheric layers where the far wings of the lines are formed.

As might be expected, the magnetic broadening effect deepens the line wings and makes the line core slightly less deep. However, such effects become noticeable only if the lines have sufficiently pronounced wings and relatively large effective Landé factors, like the C I $9603.0 \AA$ line with $g_{\mathrm{L}}=2$ (see the dot-dashed line in the top left panel of Fig. 4). Generally, the $\langle\Delta D\rangle_{\Delta \lambda}$ values represent only a fraction of one percent. Since the opacity in the line wings that is due to Zeeman broadening increases, their formation region is shifted to higher layers where the temperature is lower. At the same time, the line core opacity decreases and, hence, the formation region is shifted to deeper layers. But again, the percentage difference in the temperature is less than $0.1 \%$ (see the dot-dashed line in the bottom left panel of Fig. 4), which corresponds to a maximum temperature difference of $+5 \mathrm{~K}$ and $-1 \mathrm{~K}$, respectively, for the wings and the cores of the lines of Table 1. As a consequence, the errors of the CNO abundance determination caused by ignoring the magnetic line broadening are small.

The top right panel of Fig. 4 shows that changes $\langle\Delta D\rangle_{\Delta \lambda}$ of the line depths of the C I 5380.3,9603.0 and $10685.3 \AA$ lines due to the indirect impact of the SSD magnetic fields are noticeably larger. Interestingly, they are most pronounced in the line wings 

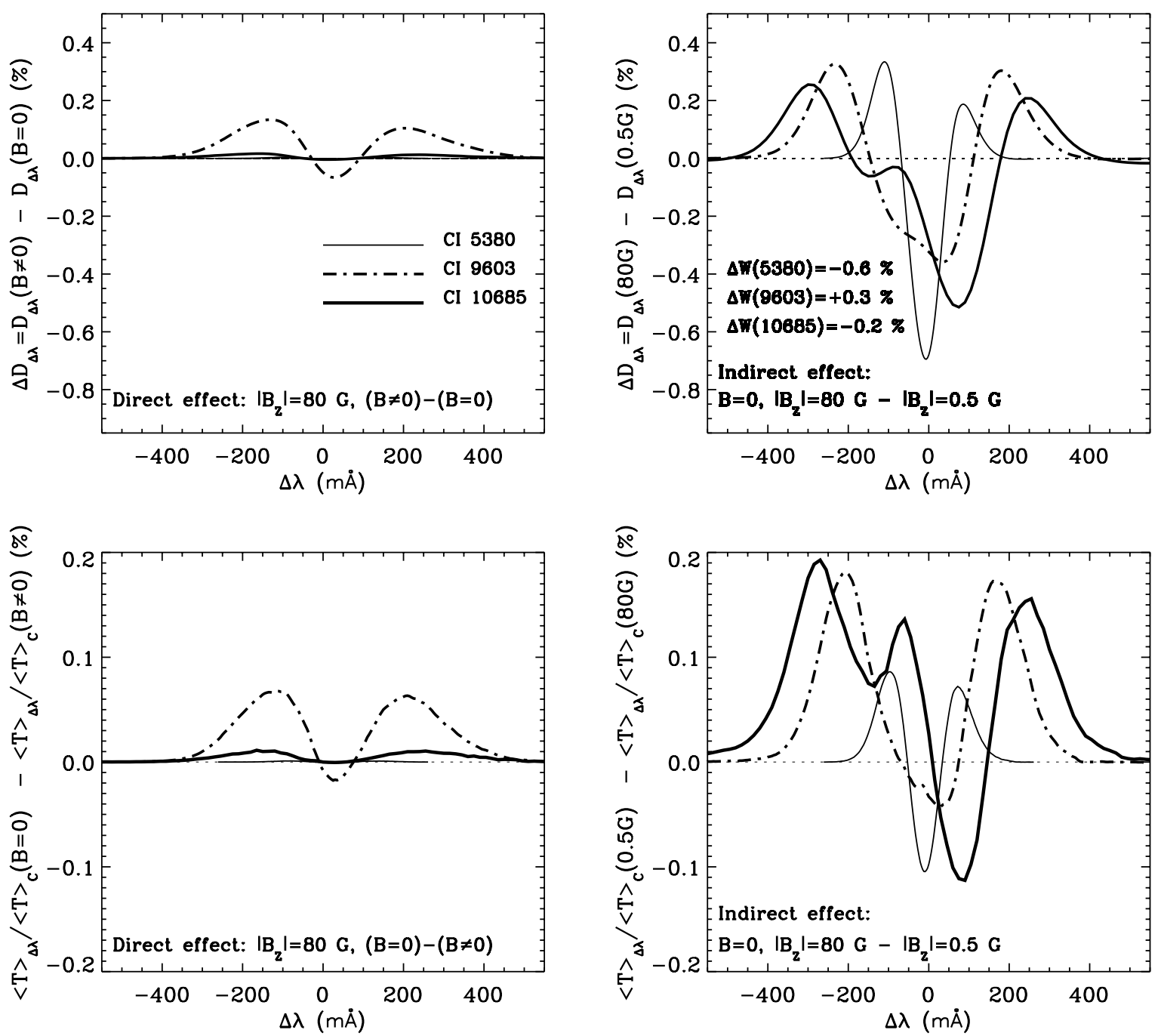

Fig. 4. Influence of direct (left panels) and indirect (right panels) effects on the C I lines $5380.3 \AA$, $9603.0 \AA$, and $10685.3 \AA$. Top panels: change $\langle\Delta D\rangle_{\Delta \lambda}$ of the line depth $\langle D\rangle_{\Delta \lambda}$ along the spatially averaged profile of each line due to Zeeman broadening (left) and due to using the $\left\langle\left|B_{z}\right|\right\rangle=80 \mathrm{G}$ snapshot instead of the $\left\langle\left|B_{z}\right|\right\rangle=0.5 \mathrm{G}$ snapshot (right). Bottom panels: change in the mean temperature $\langle T\rangle_{\Delta \lambda}$ of the formation layers of the line emerging intensity $\langle I\rangle_{\Delta \lambda}$ caused by taking into account Zeeman broadening (left) and caused by employing the $\left\langle\left|B_{z}\right|\right\rangle=0.5 \mathrm{G}$ snapshot instead of the $\left\langle\left|B_{z}\right|\right\rangle=80 \mathrm{G}$ snapshot. The temperature changes are measured in units of the mean continuum temperature $\langle T\rangle_{\mathrm{c}}$.

and in the line cores. At the same time, the $\langle\Delta D\rangle_{\Delta \lambda}$ differences for these two parts of the profiles are opposite in sign. Thus, when calculating the difference between the equivalent widths $W$ for the $\left\langle\left|B_{z}\right|\right\rangle=80 \mathrm{G}$ and the $\left\langle\left|B_{z}\right|\right\rangle=0.5 \mathrm{G}$ snapshots the contribution from the wings is compensated for by opposite contributions from the line cores. As a result, the $\Delta W$ differences are small. The top left panel of Fig. 4 shows that for the C I 5380.3, 9603.0, and $10685.3 \AA$ lines the $\Delta W$-values equal $-0.6,+0.3$, and $-0.2 \%$, respectively.

Finally, the bottom left panel shows that changes of the line depths are mainly produced by the temperature changes. Taking into account that under LTE the emerging intensity is controlled by the Planck function and that therefore the $\langle I\rangle_{\Delta \lambda}$ depends on the electron temperature of the layer where it is formed, we assume that the relative $\langle T\rangle_{\Delta \lambda} /\langle T\rangle_{\mathrm{c}}$ changes have to correlate with the $\langle\Delta D\rangle_{\Delta \lambda}$ changes of the line depth. Calculations of correlation coefficients between curves in the top and bottom right panels of Fig. 4 confirm this assumption. We found that they are rather high, being in the range between 0.7 and 0.9. This means that the indirect impact of the SSD fields is first of all caused by magnetically induced changes of the photospheric temperature.

\section{Conclusions}

The results of our investigation lead us to the following conclusions. The direct effect (through Zeeman broadening) of the SSD photospheric magnetic fields presented in the MHD snapshots by Rempel (2014) on the CNO solar abundance determination is very small. The indirect effect of these fields through magnetically induced changes of the temperature - optical depth stratification in the formation region of the $\mathrm{C} \mathrm{I}, \mathrm{N} \mathrm{I}$, and $\mathrm{O}$ I lines - is of the same order as the direct effect, but is of opposite sign. As a result, the joint (direct+indirect) impact on the determination of the solar abundances of carbon, nitrogen, and oxygen is completely negligible.

Acknowledgements. We are very grateful to M. Rempel (HAO) for kindly providing the $3 \mathrm{D}$ model atmospheres we have used in this investigation, and for helpful scientific discussions. Thanks are also due to D. Fabbian, E. Khomenko and F. Moreno-Insertis for scientific conversations. Financial support by the Spanish Ministry of Economy and Competitiveness (MINECO) through projects AYA2010-18029 (Solar Magnetism and Astrophysical Spectropolarimetry) and AYA2014-55078-P (The Solar Atmosphere: theory, computing tools and support to observations) are gratefully acknowledged. N.G. Shchukina is also grateful to the MINECO, through the 2011 Severo Ochoa Program SEV-2011-0187, which granted her a sabbatical stay at the IAC. 


\section{References}

Anders, E., \& Grevesse, N. 1989, Geochim. Cosmochim. Acta, 53, 197 Asplund, M. 2000, A\&A, 359, 755

Asplund, M., Nordlund, Å., Trampedach, R., \& Stein, R. F. 2000, A\&A, 359, 743

Asplund, M., Grevesse, N., Sauval, A. J., Allende Prieto, C., \& Kiselman, D. 2004, A\&A, 417, 751

Asplund, M., Grevesse, N., \& Sauval, A. J. 2005a, in Cosmic Abundances as Records of Stellar Evolution and Nucleosynthesis, eds. F. N. Bash, \& T. G. Barnes (San Francisco: ASP), ASP Conf. Ser., 336, 25

Asplund, M., Grevesse, N., Sauval, A. J., Allende Prieto, C., \& Blomme, R. 2005b, A\&A, 431, 693

Asplund, M., Grevesse, N., Sauval, A. J., \& Scott P. 2009, ARA\&A, 47, 481

Bahcall, J. N., Basu, S., Pinsonneault, M., and Serenelli, A. M. 2005, ApJ, 618, 1049

Basu, S., \& Antia, H. M. 2008, Phys. Rep., 457, 217

Borrero, J. M. 2008, ApJ, 673, 470

Caffau, E., Ludwig, H.-G., Bonifacio, P., et al. 2010, A\&A, 514, A92

Caffau, E., Ludwig, H.-G., Steffen, M., Freytag, B., \& Bonifacio, P. 2011, Sol Phys., 268, 255

Fabbian, D., Moreno-Insertis, F., Khomenko, E., \& Nordlund, Å. 2012, A\&A, 548, A35

Grevesse, N., \& Sauval, A.J. 1998, in Solar Composition and Its Evolution - from Core to Corona, eds. C. Frolich, M. Huber, S. K. Solanski, \& R. von Steiger (Dordrecht: Kluwer), 161

Gurtovenko, E. A., \& Kostik, R. I. 1989, Fraunhofer Spectrum and the System of Solar Oscillator Strengths (Kiev: Naukova Dumka) [in Russian]
Lambert, D. L. 1978, MNRAS, 182, 249

Maltby, P., Avrett, E. H., Carlsson, M., et al. 1986, ApJ, 306, 284

Martínez Pillet, V. 2013, Space Sci. Rev., 178, 141

Moore, C. S., Uitenbroek, H., Rempel, M., Criscuoli, S., \& Rast, M. P. 2015, ApJ, 799, 150

Pereira, T. M. D., Asplund, M., Collet, R., et al. 2013, A\&A, 554, A118

Rempel, M. 2014, ApJ, 789, 132

Sánchez Almeida, J., \& Martínez González, M. 2011, in Solar Polarization 6, eds. J. R. Kuhn, D. M. Harrington, H. Lin, et al. (San Francisco: ASP), ASP Conf. Ser., 437, 451

Scott, P., Asplund, M., Grevesse, N., Bergemann, M., \& Sauval, A. J. 2015, A\&A, 573, A26

Serenelli, A. M., Basu, S., Ferguson, J. W., \& Asplund, M. 2009, ApJ, 705, L123

Shchukina, N. G., \& Trujillo Bueno, J. 2015, A\&A, 579, A112

Shchukina, N., Sukhorukov, A., \& Trujillo Bueno, J. 2015, in Polarimetry: from the Sun to Stars and Stellar Environments, eds. K. N. Nagendra, S. Bagnulo, R. Centeno, \& M. Martínez González (Cambridge University Press), IAU Symp., 305, 368

Stein, R. F. 2012, Sol. Phys., 9, 4

Stenflo, J. O. 2013, A\&ARv, 2013, 21, 66

Trujillo Bueno, J., Shchukina, N., \& Asensio Ramos, A. 2004, Nature, 430, 326

Trujillo Bueno, J., Asensio Ramos, A., \& Shchukina, N. 2006, in Solar Polarization 4, eds. R. Casini, \& B. W. Lites (San Francisco: ASP), ASP Conf. Ser., 358, 269

Unsöld A. 1955, Physik der Sternatmosphären (Berlin: Springer Verlag), 2nd edn. 


\section{Appendix A: Table}

Table A.1. List of C I, N I, and O I lines.

\begin{tabular}{|c|c|c|c|c|c|c|}
\hline$\lambda(\AA)$ & EPL $(e V)$ & $\log g f$ & $g_{\mathrm{L}}$ & $W(\mathrm{~m} \AA)$ & $\Delta A(\mathrm{dex})$ & References \\
\hline \multicolumn{7}{|c|}{ C I lines } \\
\hline 4775.88 & 7.49 & -2.11 & 1.5 & 19.0 & +0.0053 & GK \\
\hline 4817.35 & 7.48 & -2.59 & 1.75 & 7.8 & +0.0053 & GK \\
\hline 5052.14 & 7.68 & -1.45 & 1.0 & 40.8 & +0.0048 & GK \\
\hline 5380.31 & 7.68 & -1.78 & 1.0 & 24.6 & +0.0037 & GK \\
\hline 6587.62 & 8.53 & -1.26 & 1.0 & 17.0 & +0.0017 & GK \\
\hline 7085.47 & 8.64 & -2.31 & 1.5 & 1.6 & +0.0015 & GK \\
\hline 7087.83 & 8.65 & -1.44 & 1.333 & 7.2 & +0.0017 & $\mathrm{C}$ \\
\hline 7111.45 & 8.64 & -1.35 & 0.75 & 12.1 & +0.0011 & GK \\
\hline 7113.17 & 8.64 & -0.86 & 1.125 & 28.0 & +0.0004 & GK \\
\hline 7116.97 & 8.64 & -1.11 & 1.167 & 18.5 & +0.0008 & GK \\
\hline 8335.16 & 7.68 & -0.48 & 1.0 & 115.1 & -0.0020 & GK \\
\hline 9111.80 & 7.49 & -0.29 & 1.5 & 175.0 & -0.0020 & $\mathrm{C}$ \\
\hline 9603.03 & 7.48 & -0.99 & 2.0 & 94.9 & -0.0083 & GK \\
\hline 10123.87 & 8.54 & -0.03 & 1.0 & 118.0 & -0.0016 & $\mathrm{C}$ \\
\hline 10685.34 & 7.48 & -0.27 & 0.5 & 200.0 & +0.0022 & $\mathrm{C}$ \\
\hline 10707.33 & 7.48 & -0.41 & 1.0 & 185.0 & -0.0028 & $\mathrm{C}$ \\
\hline 10729.53 & 7.49 & -0.42 & 1.333 & 182.0 & -0.0031 & $\mathrm{C}$ \\
\hline 10753.98 & 7.49 & -1.60 & 2.0 & 46.9 & -0.0059 & A05 \\
\hline 11748.22 & 8.64 & +0.38 & 0.75 & 149.0 & +0.0023 & $\mathrm{C}$ \\
\hline 11777.55 & 8.64 & -0.49 & 0.917 & 66.9 & -0.0013 & A05 \\
\hline 11848.73 & 8.64 & -0.71 & 1.333 & 42.0 & -0.0013 & $\mathrm{~L}$ \\
\hline 11892.91 & 8.64 & -0.23 & 1.0 & 80.0 & -0.0006 & $\mathrm{~L}$ \\
\hline 12549.49 & 8.85 & -0.54 & 1.5 & 59.0 & -0.0004 & A05 \\
\hline 12562.12 & 8.85 & -0.50 & 1.5 & 62.4 & -0.0005 & A05 \\
\hline 12569.04 & 8.85 & -0.59 & 1.5 & 55.9 & -0.0002 & A05 \\
\hline 12581.59 & 8.85 & -0.51 & 1.5 & 61.3 & -0.0006 & A05 \\
\hline \multicolumn{7}{|c|}{ N I lines } \\
\hline 7468.28 & 10.33 & -0.05 & 1.3 & 5.6 & +0.0020 & GK \\
\hline 8216.30 & 10.33 & +0.11 & 1.6 & 8.1 & +0.0013 & GK \\
\hline 8242.52 & 10.33 & +0.29 & 1.5 & 11.2 & +0.0008 & GK \\
\hline 8683.39 & 10.33 & +0.15 & 1.1 & 9.1 & +0.0011 & GK \\
\hline 8711.64 & 10.33 & -0.14 & 1.467 & 5.6 & +0.0012 & GK \\
\hline 8718.76 & 10.34 & -0.17 & 1.486 & 4.9 & +0.0014 & GK \\
\hline 9392.80 & 10.69 & +0.31 & 1.1 & 7.5 & +0.0015 & $\mathrm{~L}$ \\
\hline 10112.48 & 11.76 & +0.58 & 1.071 & 3.6 & +0.0028 & $\mathrm{~L}$ \\
\hline 10114.64 & 11.76 & +0.74 & 1.167 & 5.5 & +0.0026 & $\mathrm{~L}$ \\
\hline \multicolumn{7}{|c|}{ O I lines } \\
\hline 5577.34 & 1.96 & -8.01 & 2.0 & 3.2 & +0.0021 & GK \\
\hline 6156.78 & 10.74 & -0.39 & 1.167 & 5.6 & +0.0046 & GK \\
\hline 6158.17 & 10.74 & -0.42 & 1.583 & 5.2 & +0.0052 & GK \\
\hline 6300.31 & 0.00 & -9.76 & 1.25 & 4.3 & +0.0052 & GK \\
\hline 6363.78 & 0.02 & -10.13 & 0.75 & 1.8 & +0.0052 & GK \\
\hline 7771.96 & 9.14 & +0.29 & 1.333 & 79.9 & -0.0052 & GK \\
\hline 7774.17 & 9.14 & +0.14 & 1.917 & 68.8 & -0.0069 & GK \\
\hline 7775.39 & 9.14 & -0.14 & 1.75 & 52.6 & -0.0052 & GK \\
\hline 8446.76 & 9.52 & +0.01 & 1.75 & 35.2 & -0.0052 & A04 \\
\hline 9265.90 & 10.74 & +0.65 & 1.583 & 31.0 & -0.0014 & GK \\
\hline 11302.28 & 10.74 & +0.03 & 1.333 & 14.0 & +0.0002 & $\mathrm{~L}$ \\
\hline
\end{tabular}

Notes. The columns denote the wavelength $\lambda$, the excitation potential of the lower level EPL, the logarithm of the oscillator strength times the multiplicity of the lower level $\log g f$, the effective Landé factor $g_{\mathrm{L}}$, the line equivalent width $W$ measured at the solar disk center, and the abundance correction $\triangle A$ related to the joint action of the direct and indirect effects of the SSD magnetic fields. 